\title{
Femoral Rollback at High-Flexion During Squatting is Related to the Improvement of Sports Activities After Bicruciate-Stabilized Total Knee Arthroplasty: An Observational Study
}

\section{Kenichi Kono}

The University of Tokyo

Hiroshi Inui ( $\sim$ hiroshi_inu0707@yahoo.co.jp )

The University of Tokyo

Tomofumi Kage

The University of Tokyo

\section{Tetsuya Tomita}

Osaka University Graduate School of Medicine

\section{Takaharu Yamazaki}

Saitama Institute of Technology

\section{Shuji Taketomi}

The University of Tokyo

Ryota Yamagami

The University of Tokyo

Kohei Kawaguchi

The University of Tokyo

\section{Sakae Tanaka}

The University of Tokyo

\section{Research Article}

Keywords: Bicruciate-stabilized total knee arthroplasty, Sports, Patient-reported outcomes, Kinematics

Posted Date: February 17th, 2022

DOI: https://doi.org/10.21203/rs.3.rs-1205846/v1

License: (c) (i) This work is licensed under a Creative Commons Attribution 4.0 International License. Read Full License 


\section{Abstract \\ Background}

In bicruciate-stabilized total knee arthroplasty (BCS-TKA), the relationship between the postoperative kinematics and sports subscales in patient-reported outcome measures (PROMs) remains unknown. The purpose of this study was to determine the relationship between kinematics and sports subscales using the PROMs after BCS-TKA.

\section{Methods}

Sixty-one patients with severe knee osteoarthritis were examined at $13.5 \pm 7.8$ months after BCS-TKA. The patients performed squattings under single fluoroscopic surveillance in the sagittal plane. Range of motion of the knee, axial rotation of the femur relative to the tibial component, and anteroposterior (AP) translation of the medial and lateral femorotibial contact points were measured using a 2D-to-3D registration technique. In addition, the relationship between the kinematics and improvement of the sports subscales in the Knee Injury and Osteoarthritis Outcome Score (KOOS) was evaluated.

\section{Results}

In rotation angle, the femoral external rotation was observed from $0-50^{\circ}$ of flexion. The amount of femoral external rotation did not correlate with PROMs-SP. In medial AP translation, posterior translation was observed from $0-20^{\circ}$ and $80-110^{\circ}$ of flexion. The mild anterior translation was observed from $20-$ $80^{\circ}$ of flexion. Beyond $80^{\circ}$ of flexion, posterior translation was positively correlated with squatting. In lateral AP translation, posterior translation was observed from $0-20^{\circ}$ and $80-110^{\circ}$ of flexion. Beyond $80^{\circ}$ of flexion, posterior translation was positively correlated with running, jumping, twisting/pivoting, and kneeling.

\section{Conclusion}

Femoral rollback at high flexion during squatting is related to the improvement in sports activities after BCS-TKA.

\section{Background}

In bicruciate-stabilized total knee arthroplasty (BCS-TKA), the anterior and posterior cruciate ligaments are substituted, wherein the anatomical implant surface provides physiological kinematics [1]. The Journey II BCS (Smith \& Nephew, Memphis, TN, USA) is redesigned to reduce an iliotibial band traction syndrome [2]. In addition, BCS-TKA provides sufficient outcomes in activities of daily living (ADL) [1, 3, 4]. 
Recently, with the improvements in ADL after BCS-TKA, patients have been expected to participate in sports activities. In the Knee Society survey, the number of sports activities allowed after TKA has increased [5]. Furthermore, several studies reported that the return to sports or implant survivorship after TKA is favourable [6-8]. In contrast, Huch et al. reported that the prevalence of postoperative athletic activity was higher in the hip replacement group than in the knee replacement group due to greater pain relief in the hip replacement group $[5,9]$. Additionally, Mont et al. reported that the failure rate was higher in the younger and highly active patients [8].

High-flexion activities, such as exercising and gardening, have recently been classified as ADL. Thus, many patients who undergo TKA are expected to perform high-flexion activities. However, it is difficult for the patients to squat, also the limited range of motion (ROM) is negatively correlated with patient expectations [10]. Moreover, Matsushita et al. demonstrated that the improvement in ADL was related to a high satisfaction or expectation to be able to perform sports activities [11]. Therefore, the evaluation of patients' ability to perform high-flexion activities is important.

Several studies have demonstrated that the postoperative knee kinematics was related to the patientreported outcome measures (PROMs) [12-14]. Van Onsem et al. reported that the high PROM group had more pronounced posterior translation laterally from $60^{\circ}$ to $90^{\circ}$ of flexion than the low PROM group [15]. However, the relationship between the postoperative kinematics and sports subscales in PROMs remains unknown.

The objective of the current study is to clarify the relationship between kinematics and sports subscales during high-flexion activities based on the PROMs after BCS-TKA. This study hypothesised that posterior translation with flexion was related to the improvement of PROMs concerning sports (PROMs-SP) after BCS-TKA.

\section{Methods}

Sixty-one knees with severe medial osteoarthritis that underwent BCS-TKA were examined. Patients who could perform squatting safely at a natural pace after surgery were examined under single fluoroscopy surveillance in the sagittal plane according to the previously described method [14].

To estimate the spatial position and orientation of the femoral and tibial components, a 2D-to-3D registration technique was used $[16,17]$. The margin of error of the estimated relative motion between the femoral and tibial components is $\leq 0.5^{\circ}$ for rotation and $\leq 0.4^{\circ}$ for translation [16]. The following variables were evaluated: knee flexion, axial rotation of the femoral component relative to the tibial component, and anteroposterior (AP) translation of the medial and lateral femorotibial contact points. A local coordinate system at the femoral component was constructed according to previously described methods $[16,18]$. Flexion and femoral external rotation were denoted as positive values. Positive or negative values of AP translation were defined as anterior or posterior to the axes of the tibial component, respectively. The femorotibial contact point was defined as the region in which the proximity of the component surfaces was less than $0.5-\mathrm{mm}$ [14]. All data are expressed as means \pm standard deviations. 
The Knee Injury and Osteoarthritis Outcome Scores (KOOS) [19] were taken preoperatively (within 1 month) and postoperatively (at the fluoroscopic analyses) as part of PROMs. In addition, the relationship between the kinematics and the improvement of PROMs-SP was evaluated.

All patients provided written informed consent for participation. This study was approved by the concerned institutional review board. In addition, all methods were performed in accordance with the relevant guidelines and regulations.

\section{Statistical Analyses}

To analyse the data, SPSS version 25 (IBM Corp., Armonk, NY, USA), was used. Two-way analysis of variance (ANOVA) and post hoc pairwise comparisons (Bonferroni test) was used to evaluate the axial rotation and AP translation. A paired t-test was used to evaluate the improvement in PROMs-SP. The Spearman rank correlation coefficient was used to evaluate the correlation between kinematics and PROMs-SP. The level of statistical significance was set at $p<0.05$. A power analysis was performed with $G *$ Power [20] using an a error of 0.05 , and a $1-\beta$ error of 0.80 to compare the means between the two groups; a sample of 44 knees was found to be sufficient for this study.

\section{Results}

Fluoroscopic analyses were performed $13.5 \pm 7.8$ months after surgery. At the time of analysis, the mean age of the patients was $74.8 \pm 7.2$ years, the mean height was $155.0 \pm 7.8 \mathrm{~cm}$, and the mean body weight was $64.6 \pm 10.6 \mathrm{~kg}$. Of the $61 \mathrm{knees}$ included in the analysis, 9 belonged to males and 52 belonged to females.

\section{Flexion angle and rotation angle}

The knees were gradually flexed from $-2.9 \pm 6.4^{\circ}$ to $109.5 \pm 13.34^{\circ}$. In the rotation angle, the femoral component displayed an external rotation angle of $3.2 \pm 3.1^{\circ}$ relative to the tibial component from $0^{\circ}$ to $50^{\circ}$ of flexion. Beyond $50^{\circ}$ of flexion, there was no significant movement (Fig. 1).

\section{AP translation}

At the medial contact point, the femoral component showed $3.9 \pm 2.0 \mathrm{~mm}$ of posterior movements from $0^{\circ}-20^{\circ}$ of flexion, followed by $1.8 \pm 2.4 \mathrm{~mm}$ of anterior movements up to $80^{\circ}$ of flexion. Beyond $80^{\circ}$ of flexion, the femoral component showed $2.8 \pm 1.4 \mathrm{~mm}$ posterior movements (Fig. 2).

At the lateral contact point, the femoral component showed $7.7 \pm 2.8 \mathrm{~mm}$ of posterior movement from $0^{\circ}$ $-20^{\circ}$ of flexion with no significant movement up to $80^{\circ}$ of flexion. Beyond $80^{\circ}$ of flexion, a posterior movement of $3.5 \pm 1.8 \mathrm{~mm}$ was observed (Fig. 3).

\section{The improvement of PROMs-SP}


As summarised in Table 1, all function, sports, and recreational activities subscales in the KOOS improved postoperatively $(p<0.01)$.

Table 1. The improvement of patient-reported outcome measures concerning sports (PROMs-SP)

\begin{tabular}{llll} 
KOOS (Function, sports and recreational activities) & Preoperative & Postoperative & Improvement \\
\hline Squatting & $5.0 \pm 5.5$ & $11.3 \pm 6.6$ & $6.3 \pm 7.4$ \\
\hline Running & $2.6 \pm 3.7$ & $9.1 \pm 6.2$ & $6.5 \pm 6.4$ \\
\hline Jumping & $2.5 \pm 3.6$ & $8.9 \pm 6.1$ & $6.4 \pm 6.4$ \\
\hline Twisting/pivoting & $5.6 \pm 4.8$ & $13.9 \pm 6.3$ & $8.4 \pm 7.5$ \\
\hline Kneeling & $5.0 \pm 4.7$ & $11.2 \pm 6.2$ & $6.2 \pm 6.6$
\end{tabular}

Correlation between the kinematics and PROMs-SP

In the rotation angle, femoral external rotation was observed from $0^{\circ}-50^{\circ}$ of flexion. The amount of femoral external rotation did not correlate with PROMs-SP (Table 2).

Table 2. The correlation between rotation and patient-reported outcome measures concerning sports (PROMs-SP)

Flexion angle KOOS (Function, sports and recreational activities)

\begin{tabular}{lllllll} 
& & Squatting & Running & Jumping & Twisting/pivoting & Kneeling \\
\hline $0-50^{\circ}$ & rs & 0.16 & 0.16 & 0.03 & 0.22 & 0.12 \\
\cline { 2 - 6 } & p-value & 0.21 & 0.23 & 0.82 & 0.08 & 0.37
\end{tabular}

In medial AP translation, posterior translation was observed at $0-20^{\circ}$ and $80-110^{\circ}$ of flexion. The mild anterior translation was observed from $20-80^{\circ}$ to flexion. Beyond $80^{\circ}$ of flexion, posterior translation was positively correlated with squatting (Table 3 ).

Table 3. The correlation between medial anteroposterior translation and patient-reported outcome measures concerning sports (PROMs-SP) 
Flexion angle KOOS (Function, sports and recreational activities)

\begin{tabular}{lllllll} 
& & Squatting & Running & Jumping & Twisting/pivoting & Kneeling \\
\hline $0-20^{\circ}$ & rs & -0.04 & -0.23 & -0.22 & -0.02 & 0.03 \\
\cline { 2 - 7 } & p-value & 0.72 & 0.07 & 0.1 & 0.87 & 0.8 \\
\hline $20-80^{\circ}$ & rs & 0.14 & 0.12 & 0.08 & -0.09 & -0.07 \\
& p-value & 0.27 & 0.33 & 0.52 & 0.48 & 0.57 \\
\hline $80-110^{\circ}$ & rs & 0.34 & 0.14 & 0.11 & 0.21 & 0.25 \\
& p-value & 0.008 & 0.27 & 0.42 & 0.11 & 0.06
\end{tabular}

In lateral AP translation, posterior translation was observed from $0-20^{\circ}$ and to $80-110^{\circ}$ of flexion. Beyond $80^{\circ}$ of flexion, posterior translation was positively correlated with running, jumping, twisting/pivoting, and kneeling (Table 4).

Table 4. The correlation between lateral anteroposterior translation and patient-reported outcome measures concerning sports (PROMs-SP)

Flexion angle KOOS (Function, sports and recreational activities)

\begin{tabular}{lllllll} 
& & Squatting & Running & Jumping & Twisting/pivoting & Kneeling \\
\hline $0-20^{\circ}$ & rs & -0.11 & -0.23 & -0.19 & 0.06 & -0.06 \\
\cline { 2 - 6 } & p-value & 0.36 & 0.07 & 0.15 & 0.64 & 0.67 \\
$80-110^{\circ}$ & rs & 0.16 & 0.42 & 0.3 & 0.34 & 0.28 \\
& p-value & 0.23 & 0.001 & 0.02 & 0.009 & 0.03
\end{tabular}

KOOS: Knee Injury and Osteoarthritis Outcome Score

\section{Discussion}

The most important finding of this study was that the posterior translation at high flexion was correlated with the improvements in PROMs-SP, such as squatting, running, jumping, twisting/pivoting, and kneeling. In normal knees, femoral posterior rollback is observed at high flexion [21, 22]. Furthermore, Dennis et al. reported that femoral posterior movement at high flexion increased the flexion angle [23]. In addition, another study reported that the high-PROM group showed posterior femoral movement at high flexion [15]. These facts suggest that it is important to drive the femoral posterior movement at high flexion, similar to normal knees, for satisfactory sports activities after TKA. Kage et al. reported that medial and lateral AP translation did not correlate with improvements in KOOS pain, symptoms, and ADL 
[12]. Therefore, femoral posterior movement is required, especially during high flexion activities, for sports activities. Several studies have reported that the rate of return to sports (RTS) after TKA was $>70 \%[7$, 24-26]. The femoral rollback guiding designs or procedures may improve the RTS rate.

There was no significant correlation between rotation at early flexion and PROMs-SP in the current study. Kage et al. reported that at early flexion, femoral external rotation was positively correlated with improvement of the KOOS pain subscale [12]. These findings suggest that although after TKA, femoral external rotation and pain improves, it may be difficult to improve high flexion activities such as sports.

In this study, we observed a mild femoral external rotation with flexion. This rotational kinematic pattern was similar to that reported in previous studies $[1,12,14,27]$. One of these previous studies reported that the lower PROM group in BCS-TKA indicated excessive femoral external rotation with flexion [14]. In the current study, the PROMs-SP improved in all subscales. Therefore, after BCS-TKA, excessive femoral external rotation may not be needed to improve sports activities.

The AP translation in this study was similar to that in previous studies $[1,12,14,27]$. In normal knees, medial pivot motion was observed during early flexion [21]. In other words, no medial posterior translation was observed during early flexion. In contrast, the knees after BCS-TKA showed medial posterior translation during early flexion. Ishibashi et al. reported that medial posterior translation during early flexion was due to anterior post-cam engagement [27]. To achieve normal-like kinematics, further improvement of the post-cam mechanism might be required.

In this study, after BCS-TKA, all sports subscales improved. Vielgut et al. demonstrated that postoperative sports ability was correlated with preoperative activity [28]. Additionally, Ho et al. demonstrated that the RTS rate between TKA and unicompartmental knee arthroplasty did not differ under the same preoperative patient characteristics [29]. Thus, preoperative activity may have influenced the improvement of PROMs-SP.

This study had several limitations. First, in the current study, only squatting was measured. The previous studies reported that knee kinematics differ depending on the type of activities [21,30]. Therefore, the kinematic effect during other activities might be different from squatting. Second, the squatting motion was measured only one movement repetition per patient because of ethical reasons to reduce patients' $X$ ray exposure.

\section{Conclusion}

The femoral rollback at high flexion during squatting is related to the improvement of sports activities after BCS-TKA.

\section{List Of Abbreviations}

ADL activities of daily living 
ANOVA analysis of variance

AP anteroposterior

BCS-TKA bicruciate-stabilized total knee arthroplasty

DKB deep knee bending

KOOS Knee Injury and Osteoarthritis Outcome Score

PROMs patient-reported outcome measures

PROMs-SP patient-reported outcome measures concerning sports

ROM range of motion

RTS return to sports

TKA total knee arthroplasty

\section{Declarations}

Ethics approval and consent to participate: This study was performed in accordance with the principles of the Declaration of Helsinki. Approval was granted by the University of Tokyo Institutional Ethics Review Board [number 10462-(1)]. Informed consent was obtained from all participants included in the study.

Consent for publication: The patients signed informed consent forms for the publication of their data and photographs.

\section{Availability of data and materials:}

The datasets used during the current study are available from the corresponding author on reasonable request. The data used during the current study could not be deposited because the patients did not approve the permanent availability of data.

Competing interests: None.

\section{Funding}

This research did not receive any specific grant from funding agencies in the public, commercial, or notfor-profit sectors.

Author contributions: Kenichi Kono helped perform the study and formal analysis and wrote the manuscript. Hiroshi Inui and Tomofumi Kage carried out data curation and conceived the study. Takaharu Yamazaki, Ryota Yamagami, and Kohei Kawaguchi provided technical assistance. Tetsuya 
Tomita, Shuji Taketomi, and Sakae Tanaka provided general support. All authors read and approved the final manuscript.

Acknowledgement: Not applicable

\section{References}

1. Kono K, Inui H, Tomita T, Yamazaki T, Taketomi S, Sugamoto K, et al. Bicruciate-stabilised total knee arthroplasty provides good functional stability during high-flexion weight-bearing activities. Knee Surg Sports Traumatol Arthrosc. 2019;27:2096-103 https://doi.org/10.1007/s00167-019-05375-9.

2. Luyckx L, Luyckx T, Bellemans J, Victor J. Iliotibial band traction syndrome in guided motion TKA. A new clinical entity after TKA. Acta Orthop Belg. 2010;76:507-12.

3. Kaneko T, Kono N, Mochizuki Y, Hada M, Toyoda S, Musha Y. Bi-cruciate substituting total knee arthroplasty improved medio-lateral instability in mid-flexion range. J Orthop. 2017;14:201-6. https://doi.org/10.1016/j.jor.2016.12.005.

4. Inui $\mathrm{H}$, Taketomi S, Yamagami R, Kono K, Kawaguchi K, Takagi K, et al. Comparison of intraoperative kinematics and their influence on the clinical outcomes between posterior stabilized total knee arthroplasty and bi-cruciate stabilized total knee arthroplasty. Knee. 2020;27:1263-70. https://doi.org/10.1016/j.knee.2020.06.008.

5. Healy WL, Sharma S, Schwartz B, lorio R. Athletic activity after total joint arthroplasty. J Bone Joint Surg Am. 2008;90:2245-52. https://doi.org/10.2106/JBJS.H.00274.

6. Crawford DA, Adams JB, Hobbs GR, Berend KR, Lombardi AV, Jr. Higher activity level following total knee arthroplasty is not deleterious to mid-term implant survivorship. J Arthroplasty. 2020;35:11620. https://doi.org/10.1016/j.arth.2019.07.044.

7. Witjes S, Gouttebarge V, Kuijer PP, van Geenen RC, Poolman RW, Kerkhoffs GM. Return to sports and physical activity After total and unicondylar knee arthroplasty: A systematic review and metaanalysis. Sports Med. 2016;46:269-92. https://doi.org/10.1007/s40279-015-0421-9.

8. Mont MA, Marker DR, Seyler TM, Jones LC, Kolisek FR, Hungerford DS. High-impact sports after total knee arthroplasty. J Arthroplasty. 2008;23;Suppl 1:80 - 4.

https://doi.org/10.1016/j.arth.2008.04.018.

9. Huch K, Müller KA, Stürmer T, Brenner H, Puhl W, Günther KP. Sports activities 5 years after total knee or hip arthroplasty: The Ulm osteoarthritis Study. Ann Rheum Dis. 2005;64:1715-20. https://doi.org/10.1136/ard.2004.033266.

10. Matsuda S, Kawahara S, Okazaki K, Tashiro Y, Iwamoto Y. Postoperative alignment and ROM affect patient satisfaction after TKA. Clin Orthop Relat Res. 2013;471:127-33. https://doi.org/10.1007/s11999-012-2533-y.

11. Matsushita Y, Hamai S, Okazaki K, Murakami K, Ma Y, Kiyohara M, et al. Recreational sports, workout and gym activities after total knee arthroplasty: Asian cohort study. J Orthop. 2019;16:41-4. https://doi.org/10.1016/j.jor.2018.12.002. 
12. Kage $T$, Inui $H$, Tomita T, Yamazaki T, Taketomi $S$, Yamagami $R$, et al. The association between in vivo knee kinematics and patient-reported outcomes during squatting in Bicruciate-stabilized total knee arthroplasty. J Knee Surg. 2021. https://doi.org/10.1055/s-0041-1723763.

13. Pizza N, Di Paolo S, Zinno R, Marcheggiani Muccioli GM, Agostinone P, Alesi D, et al. Overconstrained kinematic of the medial compartment leads to lower clinical outcomes after total knee arthroplasty. Knee Surg Sports Traumatol Arthrosc. 2021 https://doi.org/10.1007/s00167-02006398-3.

14. Kono K, Inui H, Tomita T, Yamazaki T, Taketomi S, Yamagami R, et al. The higher patient-reported outcome measure group had smaller external rotation of the femur in bicruciate-stabilized total knee arthroplasty. Knee Surg Sports Traumatol Arthrosc. 2021 https://doi.org/10.1007/s00167-02106577-w.

15. Van Onsem S, Verstraete M, Van Eenoo W, Van Der Straeten C, Victor J. Are TKA kinematics During closed kinetic chain exercises associated with patient-reported outcomes? A preliminary analysis. Clin Orthop Relat Res. 2020;478:255-63. https://doi.org/10.1097/CORR.0000000000000991.

16. Yamazaki T, Watanabe T, Nakajima Y, Sugamoto K, Tomita T, Yoshikawa H, et al. Improvement of depth position in 2-D/3-D registration of knee implants using single-plane fluoroscopy. IEEE Trans Med Imaging. 2004;23:602-12. http://ieeexplore.ieee.org/ielx5/42/28807/01295080.pdf? $\mathrm{tp}=$ \&arnumber $=1295080$ \&isnumber $=28807$.

17. Yamazaki T, Watanabe T, Nakajima Y, Sugamoto K, Tomita T, Maeda D, et al. Visualization of femorotibial contact in total knee arthroplasty using X-ray fluoroscopy. Eur J Radiol. 2005;53:84-9. https://doi.org/10.1016/j.ejrad.2003.09.018.

18. Shimizu N, Tomita T, Yamazaki T, Yoshikawa H, Sugamoto $K$. The effect of weight-bearing condition on kinematics of a high-flexion, posterior-stabilized knee prosthesis. J Arthroplasty. 2011;26:1031-7. https://doi.org/10.1016/j.arth.2011.01.008.

19. Nakamura N, Takeuchi R, Sawaguchi T, Ishikawa H, Saito T, Goldhahn S. Cross-cultural adaptation and validation of the Japanese Knee Injury and Osteoarthritis Outcome Score (KOOS). J Orthop Sci. 2011;16:516-23. https://doi.org/10.1007/s00776-011-0112-9.

20. Faul F, Erdfelder E, Buchner A, Lang AG. Statistical power analyses using G*Power 3.1: Tests for correlation and regression analyses. Behav Res Methods. 2009;41:1149-60. https://doi.org/10.3758/BRM.41.4.1149.

21. Kono K, Tomita T, Futai K, Yamazaki T, Tanaka S, Yoshikawa $H$, et al. In vivo three-dimensional kinematics of normal knees during different high-flexion activities. Bone Joint J. 2018;100-B:50 - 5 . https://doi.org/10.1302/0301-620X.100B1.BJJ-2017-0553.R2.

22. Hill PF, Vedi V, Williams A, Iwaki H, Pinskerova V, Freeman MA. Tibiofemoral movement 2: The loaded and unloaded living knee studied by MRI. J Bone Joint Surg Br. 2000;82:1196-8. http://bjj.boneandjoint.org.uk/content/jbjsbr/82-B/8/1196.full.pdf.

23. Dennis DA, Komistek RD, Mahfouz MR, Haas BD, Stiehl JB. Multicenter determination of in vivo kinematics after total knee arthroplasty. Clin Orthop Relat Res. 2003;416:37-57. 
https://doi.org/10.1097/01.blo.0000092986.12414.b5.

24. Hanreich C, Martelanz L, Koller U, Windhager R, Waldstein W. Sport and physical activity following primary total knee arthroplasty: A systematic review and meta-analysis. J Arthroplasty. 2020;35:2274-2285.e1. https://doi.org/10.1016/j.arth.2020.04.013.

25. Bradbury N, Borton D, Spoo G, Cross MJ. Participation in sports after total knee replacement. Am J Sports Med. 1998;26:530-5. https://doi.org/10.1177/03635465980260041001.

26. Hepperger C, Gföller P, Abermann E, Hoser C, Ulmer H, Herbst E, et al. Sports activity is maintained or increased following total knee arthroplasty. Knee Surg Sports Traumatol Arthrosc. 2018;26:1515-23. https://doi.org/10.1007/s00167-017-4529-3.

27. Ishibashi T, Tomita T, Yamazaki T, Tsuji S, Yoshikawa H, Sugamoto K. Kinematics of bicruciate and posterior stabilized total knee arthroplasty during deep knee flexion and stair climbing. J Orthop Res. 2021;39:1262-70. https://doi.org/10.1002/jor.24773.

28. Vielgut I, Leitner L, Kastner N, Radl R, Leithner A, Sadoghi P. Sports Activity after Low-contact-stress total knee arthroplasty - A long term follow-up study. Sci Rep:24630. Sci Rep. 2016;6:24630. https://doi.org/10.1038/srep24630.

29. Ho JC, Stitzlein RN, Green CJ, Stoner T, Froimson MI. Return to Sports Activity following UKA and TKA. J Knee Surg. 2016;29:254-9. https://doi.org/10.1055/s-0035-1551835.

30. Kono K, Inui H, Tomita T, Yamazaki T, Taketomi S, Tanaka S: In Vivo Kinematics of BicruciateRetaining Total Knee Arthroplasty with Anatomical Articular Surface under High-Flexion Conditions. J Knee Surg 2021; 34:452-459. http://doi.org/10.1055/s-0039-1696959.

\section{Figures}

degree

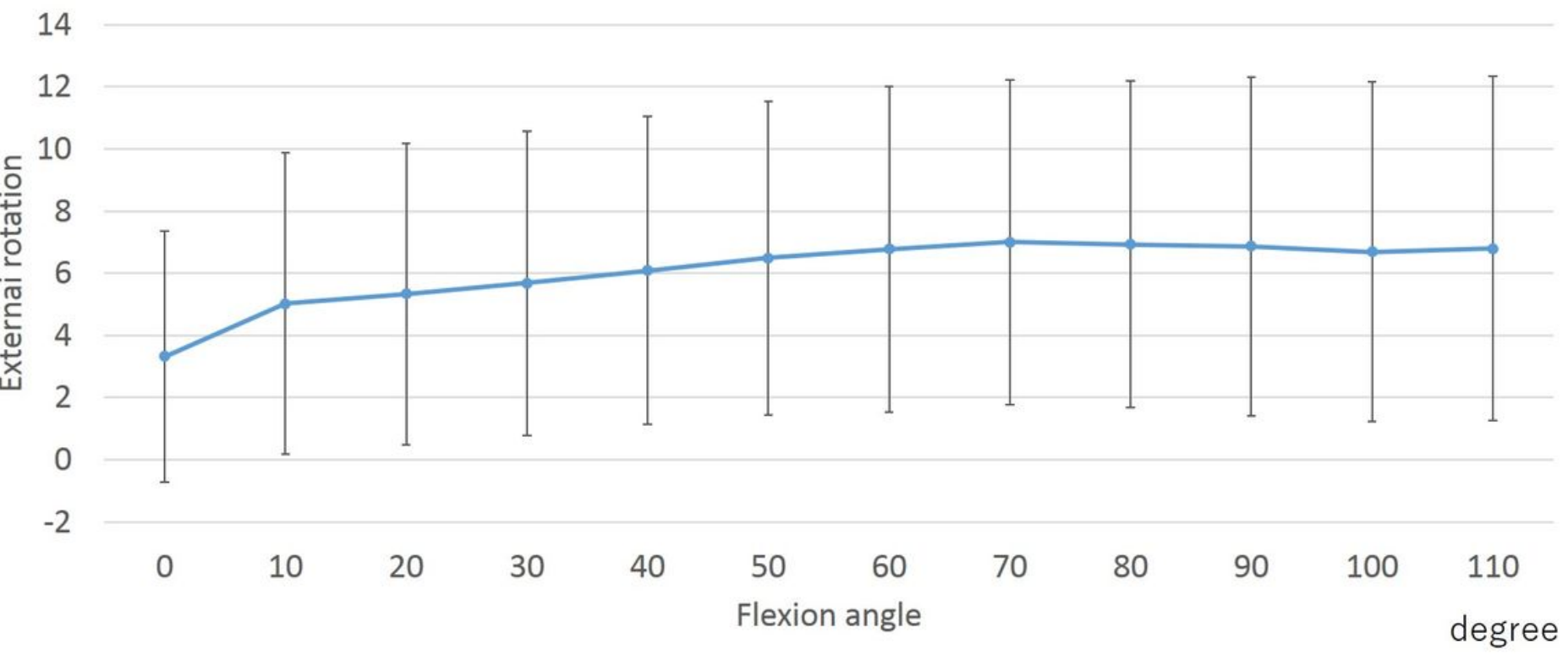

Figure 1 
Axial rotation angle

$\mathrm{mm}$

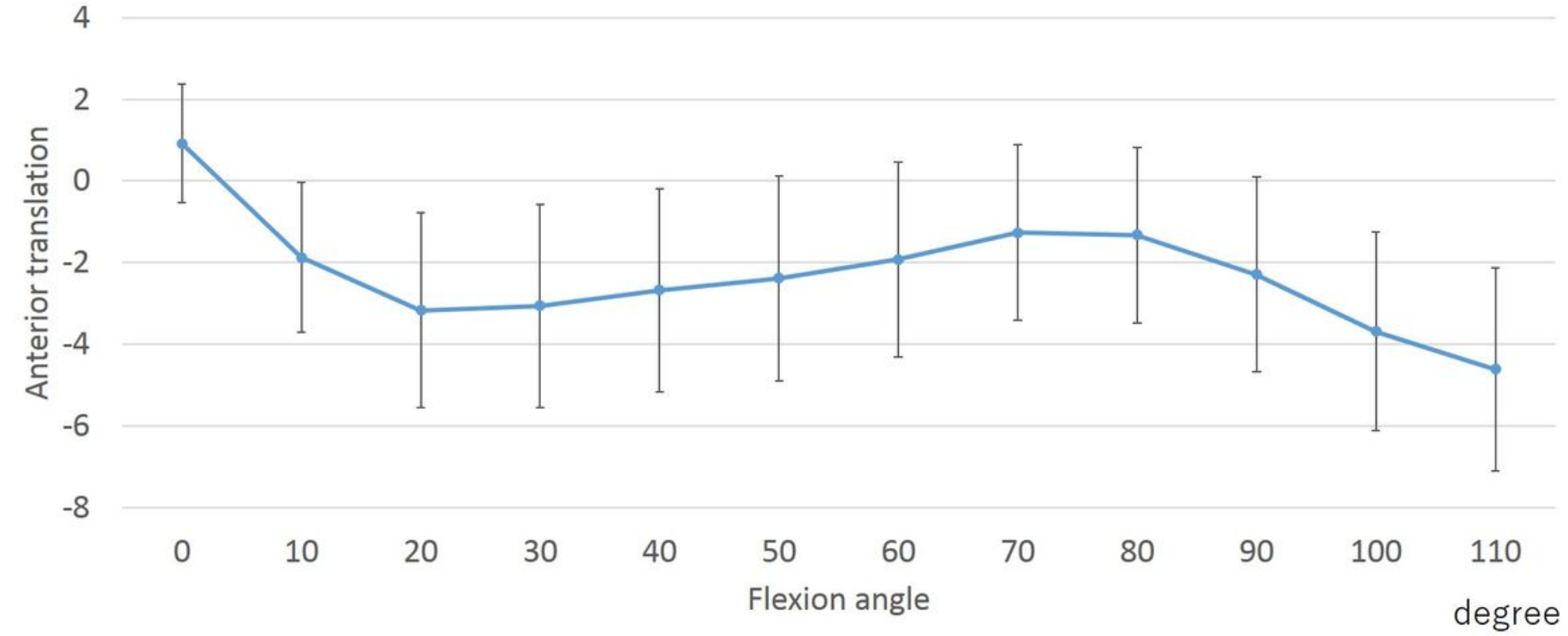

Figure 2

Medial anteroposterior translation

$\mathrm{mm}$

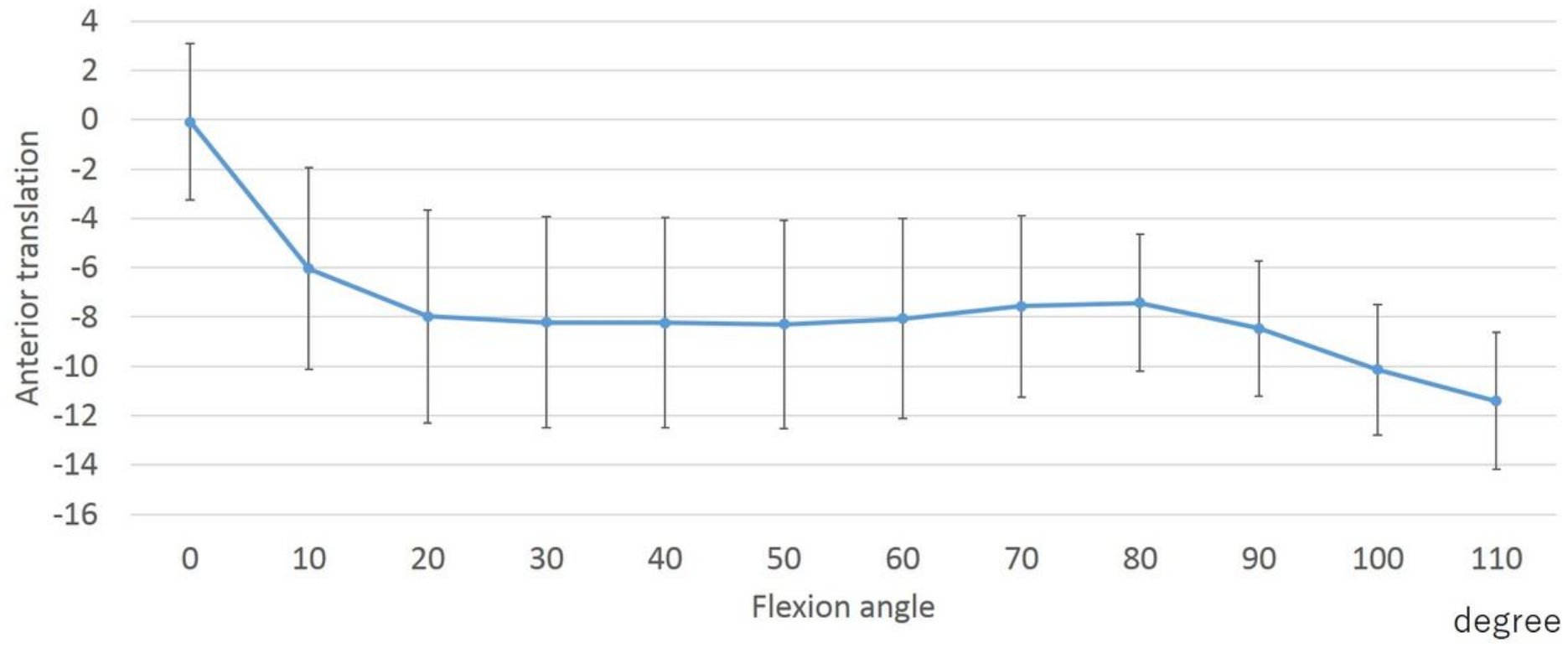

Figure 3

Lateral anteroposterior translation 